\title{
User Behaviour Profiling in Cloud using One Class SVM: A Review
}

\author{
Vijaya Lakshmi Paruchuri ${ }^{1}$, S. Suresh Babu ${ }^{2}$, P S V S Sridhar ${ }^{1}$, Debnath \\ Bhattacharyya $^{3}$ and Hye-Jin Kim ${ }^{4}$ \\ ${ }^{1}$ Department of Computer Science and Engineering, \\ KL University, Vaddeswaram, AP, 522502, India \\ \{parchuri.vijaya, psvssridhar\}@gmail.com \\ ${ }^{2}$ Department of Information Technology, VFSTR University, \\ Vadlamudi-522213, Guntur, India \\ suresh.satukumati@gmail.com \\ ${ }^{3}$ Department of Computer Science and Engineering, \\ Vignan's Institute of Information Technology \\ Visakhapatnam-530049, India \\ debnathb@gmail.com \\ ${ }^{4}$ Sungshin Women's University, \\ 2, Bomun-ro 34da-gil, \\ Seongbuk-gu, Seoul, Korea \\ hyejinaa@daum.net \\ (Corresponding Author)
}

\begin{abstract}
Distributed computing guarantees to on a very basic level change the way we utilize PCs and get to and store our own specific and business data. With these new registering and correspondences models develop new data security challenges. Existing information security structures, for instance, encryption have fizzled in imagining information theft strikes, particularly those executed by an insider to the cloud supplier. We propose a substitute methodology for securing information in the cloud utilizing adversarial mimic improvement. We screen data access in the cloud and perceive unpredictable data access outlines. Right when unapproved access is suspected and after that confirmed using test questions, we dispatch a disinformation strike by giving back a considerable measure of fake information to the attacker. This secures against the misuse of the customer's real data. Trials coordinated in a neighbor-hood archive setting give confirmation this technique may give unprecedented levels of customer data security in a Cloud space.
\end{abstract}

Keywords: Distributed computing, User Behavior Profiling, Support Vector Machine

\section{Introduction}

Cloud computing is the collection of various services over the (World Wide Web) WWW. Businesses people and individuals use software and hardware features available in the cloud which are managed by third parties. Cloud model establishes a medium to access the information along with computer resources over a network connection. A shared pool of resources, with memory allocation for data, higher CPU power, and user applications was being provided by cloud [1]. 
When your photos are stored on online, a cloud computing service is made used. In order to use it, for example, an online invoicing service instead to update the in-house one which was being used for many years, that online invoicing service is a cloud computing service. Cloud computing can be defined as the utility of remote servers across the internet to store, process, and maintain the data over many computers. Storing of data on your hard drive or updating applications on your requirements, one can use a service over the Internet, at some other location, for accessing from any point irrespective of the system or hard drive in which the data is stored. Privacy hazards may come into existence due to storing information in cloud.

\section{Related Work}

The principle reason for this paper is to actualize a superior security highlight called Fog Computing in the current cloud administrations where in the arrangement of approved and unapproved exercises result in location of disguising action in the framework. Late patterns have seen progressions in both offices in cloud and cloud information burglaries. Security is constantly considered as the need particularly in substantial scale distributed computing. There has been a critical ascent in the assaults against cloud security administrations. The assaults fundamentally are characterized into outcast and insider assaults, the last one being the most unsafe. The issue lies in the accompanying connections [2].

1) Detecting the insider assault.

2) Limiting the harm because of information robbery.

3) Locate the passageway of assault.

\subsection{Evidence for the Problem}

Obama's Twitter secret key uncovered after French programmer captured for breaking into U.S. president's record. Twitter clients make somewhere in the range of 50million messages for every day, with numerous sent by prominent VIPs.

\subsection{User Behavior Profiling}

Customers of a PC are familiar with the archives on that system and where they are discovered [3]. Any journey for specific records is obligated to be centered around and compelled. An impostor, then again, who gets to be familiar with the setback's system illegitimately, is implausible to be familiar with the structure and substance of the record system. Their request is inclined to be expansive and untargeted. In perspective of this key supposition, we profiled customer look lead and made customer models arranged with a one-class showing framework, to be particular one-class support vector machines. The criticalness of using one-class exhibiting originates from the limit of building a classifier without sharing data from particular customers. The protection of the client and their information is along these lines saved [4].

We screen for anomalous pursuit practices that show deviations from the client benchmark. As indicated by our presumption, such deviations flag a potential masquerade assault. Profiling Search Behavior the USB sensor distinguishes anomalous client seek conduct in the wake of profiling client activities and shaping a benchmark of pursuit conduct using irregularity discovery strategies. At that point it screens for anomalous pursuit practices that show substantial deviations from the gauge. Such deviations flag a potential masquerade assault.

The sensor assembles a One-Class Support Vector Machine (OCSVM) model that models the client's hunt conduct [5]. Vectors with three pursuit related components are 
removed for every two moment time of client movement. The pursuit behavior related elements are:

2.2.1. Number of computerized hunt related activities: Specific areas of the Windows registry, particular Dynamic Link Libraries (DLLs), access to particular record documents, what's more, particular projects, especially desktop hunt instruments, are associated with framework seeking. The aggregate number of these hunt related occasions are displayed per 2-minute age.

2.2.2. Number of record touches: Any documents bring, read, compose, or duplicate activity results into stacking the record into memory. The quantity of times documents are touched and stacked into memory by any procedure inside of every 2-minute age is utilized as a component.

The DDA (Decoy Document Deployment) sensor distinguishes when distraction records are being perused, replicated, or zipped [6]. When the distraction record is stacked into memory by any application or process, the sensor starts a confirmation capacity, which checks whether the document is typical or a distraction by registering a HMAC(Hash Message Authentication Code) taking into account every one of the substance of that record and contrasting it with the one inserted inside of the report.

\subsection{Decoy Technology}

We set traps inside of the record framework. The traps are distraction documents downloaded from a Fog registering website, a computerized administration that offers a few sorts of fake reports, for example, assessment form frames, restorative records, financial records, e-cove receipts, and so on.. The imitation documents are downloaded by the honest to goodness client and set in very obvious areas that are not prone to bring about any obstruction with the typical client exercises on the framework.

An impostor, who is not acquainted with the document framework and its substance, is prone to get to these imitation records, on the off chance that he or she is in quest for touchy data, for example, the lure data inserted in these fake documents. In this manner, checking access to the imitation documents ought to flag masquerade action on the framework.

The bait records convey a keyed-Hash Message Authentication Code (HMAC), which is covered up in the header segment of the report. The HMAC is figured over the document's substance utilizing a key one of a kind to every client. At the point when a fake report is stacked into memory, we check whether the archive is an imitation record by figuring a HMAC in light of the considerable number of substance of that record. We contrast it and HMAC installed inside of the record. On the off chance that the two HMACs match, the report is considered a distraction and a caution is issued. The preferences of setting baits in a document framework are three-fold:(1) the identification of masquerade movement (2) the perplexity of the assailant and the extra expenses caused to recognize genuine from counterfeit data, and (3) the discouragement impact which, albeit difficult to quantify, assumes a noteworthy part in averting masquerade action by danger loath aggressors.

Decoys usages are Effective: The principle motivation behind our utilization of fakes is to recognize impostor assaults. While non-obstruction with authentic clients' exercises is alluring, distractions would be futile on the off chance that they neglect to draw in impostors by being alluring and obvious. The outcomes propose that no less than one access to a bait record was identified by the DDA sensor for each impostor, paying little heed to the quantity of distractions planted in the record framework. This discovering demonstrates that all around put imitations can be exceptionally viable for masquerade identification. At the point when consolidated with other interruption 
identification strategies, they could possibly give considerably more successful and precise identifiers.

\subsection{Imitation Placement is Important}

The goal is to recognize the bait record areas that would be less meddling with the typical action of the honest to goodness client, while being obvious to potential aggressors. While the trials have not been led on the same framework, and the imitation document areas fluctuate by typical client (modified for their own particular non-meddling utilization of the framework), we contend that the aggregate results give ground to correlation, as we have checked the utilization for around 7 days all things considered for every client, for a sum of 52 clients.

\subsection{Combining the User Behavior Profiling and Decoy Technology}

The relationship of pursuit conduct peculiarity recognition with trap-based distraction documents ought to give more grounded confirmation of wrongdoing, and in this manner enhance an identifier's precision. We estimate that recognizing strange hunt operations performed preceding a clueless client opening a bait document will certify the suspicion that the client is in reality mimicking another casualty client. This situation covers the risk model of illegitimate access to Cloud information. Moreover, an unplanned opening of a fake document by a honest to goodness client may be perceived as a mischance if the hunt conduct is not considered strange. As such, recognizing unusual hunt and distraction traps together may make an extremely successful masquerade discovery framework. Consolidating the two methods enhances location precision.

\section{Support Vector Machines}

SVMs are set of related supervised learning methods used for classification and regression. They belong to a family of generalized linear classification. A unique property of SVM will be, SVM all the while minimize the experimental order mistake and boost the geometric edge. So SVM called Maximum Margin Classifiers. SVM depends on the Structural Risk Minimization (SRM). SVM map input vector to a higher dimensional space where a maximal separating hyperplane is built. Two parallel hyperplanes are built on each side of the hyperplane that different the information. The separating hyperplane is the hyperplane that maximize the separation between the two parallel hyperplanes [7].

A SVM model is a representation of the samples as focuses in space, mapped so that the illustrations of the different classes are separated by a reasonable crevice that is as wide as would be prudent. New cases are then mapped into that same space and anticipated to have a place with a classification.

A support vector machine constructs a hyperplane or set of hyperplanes in or infinite-dimensional space, which can be used for classification, regression, or other tasks. Intuitively, a good separation is achieved by the hyperplane that has the largest distance to the nearest training-data point of any class (so-called functional margin), since in general the larger the margin the lower the generalization error of the classifier. Architecture of SVM is shown below. 


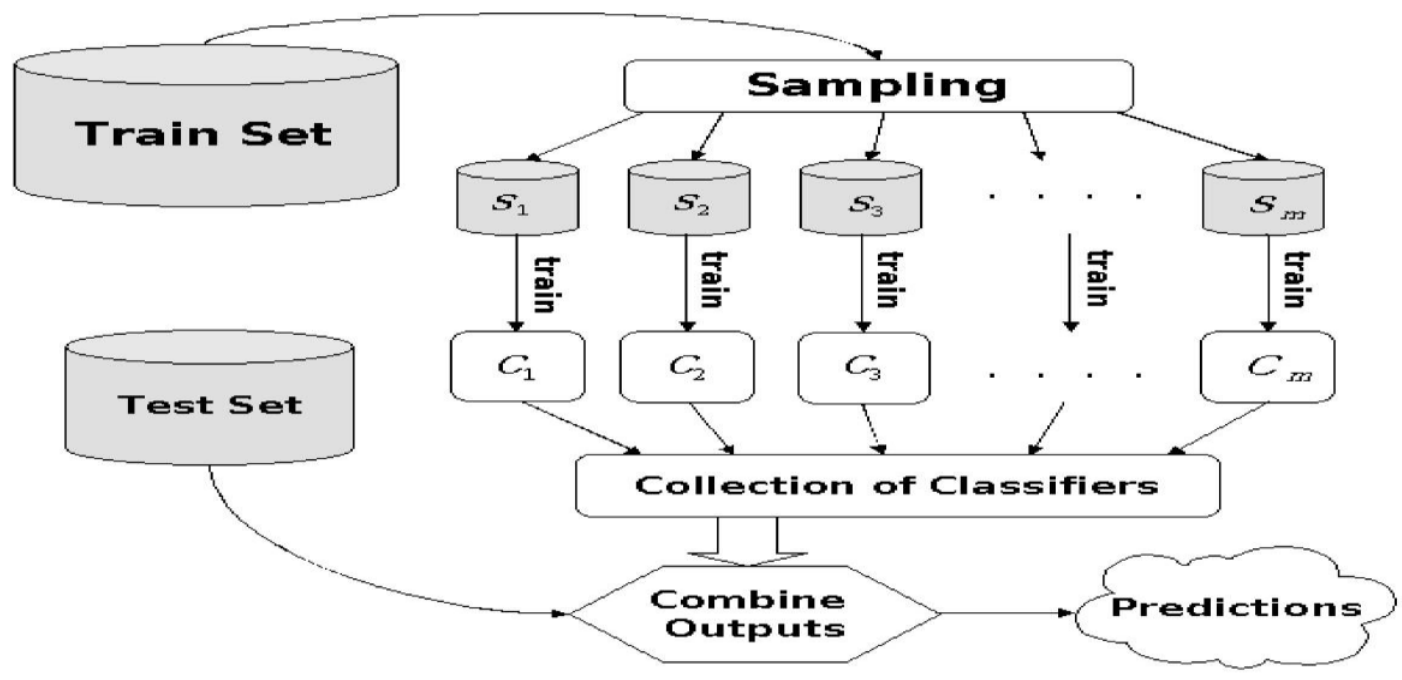

Figure 1. Typical SVM Architecture

In Figure 1, an important concept in supervised machine learning is training error vs. testing error. Since we are generally less interested in how our model works on the data it was trained on than how it works on new data, we need to create a test set (sometimes also called a holdout set). MLI provides a function to split up your data randomly into train set vs. test set. By default, it will make the training set $90 \%$ of your input data, and the testing set the remaining $10 \%$.

The SVM takes after a target capacity which helps in choosing the hyper plane that isolates orders the articles. For Example: $y=\min (1 / 2(\omega \tau) * \omega+c ̧ * \Sigma i)$

The capacity is as a comparison for a straight line $y=m x+c$. They investigate information and rearrange designs for order and relapse examine.

\subsection{One Class SVMs}

In contrast to traditional SVMs, One Class SVMs attempt to learn a decision boundary that achieves the maximum separation between the points and the origin. A One Class SVM uses an implicit transformation function $\varphi(\bullet)$ characterized by the to project the data into a higher dimensional space. The algorithm then learns the decision boundary (a hyperplane) that isolates most of the data from the origin. Just a little part of data focuses are permitted to lie on the opposite side of the decision boundary, Those data points are considered as outliers [8].

\section{Detecting Insider Attacks}

\subsection{Technical Controls}

Insider Danger Control: Utilizing Written falsification Identification Calculations to Forestall Information Exhilaration in close continuous. In associations with access to the web, the potential for information spillage is ever present. The insider risk control depicted in this specialized note can screen web solicitation activity for content based information exfiltration endeavors and square them continuously. Utilizing this control can offer an association some assistance with protecting content based licensed innovation, including source code archives.

As a feature of the copyright infringement discovery control, the Insider Risk group offers two control frameworks code tests: 
- WebDLPIndexer, a Java specialists, helps with the execution of the group's information misfortune avoidance (DLP) control

- WebDLP Customer advances active web solicitations to the WebDLPIndexer[9] specialists for correlation against a record of licensed innovation.

\subsection{Effectiveness of a Pattern for Preventing Theft by Insiders}

Since 2001, scientists at the CERT Insider Risk Center have recorded pernicious insider action by looking at media reports and court transcripts and leading meetings with the United States Mystery Administration, casualties' associations, and indicted criminals [10]. Among the more than 700 insider risk cases that we've archived, our investigation has distinguished more than 100 classes of shortcomings in frameworks, procedures, individuals or innovations that permitted insider dangers to happen.

\section{Conclusion}

Insider perils can speak to a bona fide security threat to associations. They can be brought on by some person who is purposely toxic, as Sony found, or it can be something as clear as some individual opening an association stacked with malware that licenses untouchables the opportunity to take information. Once unapproved data access or presentation is suspected, and later confirmed, with test questions for instance, we immerse the poisonous insider with fake information in order to debilitate the customer's bona fide data. Such preventive assaults that rely on upon disinformation advancement could give remarkable levels of security in the Cloud and in interpersonal organizations. Masquerade assaults represent a genuine PC security issue. Earlier work concentrated on professional ling clients. We lessened false positives by $36 \%$ over the best results reported in writing to date with a $99.94 \%$ masquerade identification rate with just $0.77 \%$ of false positives, the best results accomplished in the writing in this way.

\section{References}

[1] Y. A. A. S. Aldeen, M. Salleh and M. A. Razzaque, "A Survey Paper on Privacy Issue in Cloud Computing", Research Journal of Applied Sciences, Engineering and Technology, vol. 10, no. 3, (2015), pp. 328-337.

[2] S. J. Stolfo, M. B. Salem and A. D. Keromytis, "Fog computing: Mitigating insider data theft attacks in the cloud", IEEE Symposium on In Security and Privacy Workshops (SPW), (2012), pp. 125-128.

[3] W. N. Bhukya, and S. K. Banothu, "Investigative behavior profiling with one class SVM for computer forensics", Multi-disciplinary Trends in Artificial Intelligence, Springer Berlin Heidelberg, (2011), pp. 373-383.

[4] J. Nayak, B. Naik and H. S. Behera, "A Comprehensive Survey on Support Vector Machine in Data Mining Tasks: Applications \& Challenges", International Journal of Database Theory and Application, vol. 8, no. 1, (2015), pp. 169-186.

[5] J. Muñoz-Marí, F. Bovolo, L. Gómez-Chova, L. Bruzzone and G. Camp-Valls, "Semisupervised one-class support vector machines for classification of remote sensing data", IEEE Transactions on Geoscience and Remote Sensing, vol. 48, no. 8, (2010), pp. 3188-3197.

[6] I. Sudha, A. Kannaki and S. Jeevidha, "Alleviating Internal Data Theft Attacks by Decoy Technology in Cloud", International Journal of Computer Science and Mobile Computing, vol. 3, no. 3, (2014), pp. 217-222.

[7] J. Nayak, B. Naik and H. S. Behera, "A Comprehensive Survey on Support Vector Machine in Data Mining Tasks: Applications \& Challenges", International Journal of Database Theory and Application, vol. 8, no. 1, (2015), pp. 169-186.

[8] M. Amer, M. Goldstein and S. Abdennadher, "Enhancing one-class support vector machines for unsupervised anomaly detection", Proceedings of the ACM SIGKDD Workshop on Outlier Detection and Description, ACM, (2013), pp. 8-15.

[9] T. Lewellen, G. J. Silowash and D. L. Costa, "Insider Threat Control: Using Plagiarism Detection Algorithms to Prevent Data Exfiltration in Near Real Time", (2013).

[10] A. P. Moore, "Effectiveness of a Pattern for Detecting Intellectual Property Theft by Departing Insiders", (2012). 